\title{
GENERALIZED THEORY OF ELECTRICAL CONDUCTIVITY AND CURRENT NOISE FOR DISCONTINUOUS METAL FILMS OVER A WIDE RANGE OF TEMPERATURES
}

\author{
M. CELASCO \\ Istituto di Fisica Generale dell'Università di Torino, and G.N.S.M. (U.R.24) Torino (Italy).
}

A. MASOERO

Istituto Elettrotecnico Nazionale Galileo Ferraris di Torino, and G.N.S.M. (U.R.24) Torino (Italy).

P. MAZZETTI

Istitu to di Fisica Sperimentale del Politecnico di Torino, and G.N.S.M. (U.R.24) Torino (Italy).

and

\begin{abstract}
A. STEPANESCU
Istituto Elettrotecnico Nazionale Galileo Ferraris di Torino, and Gruppo Nazionale di Struttura della Materia (G.N.S.M. - U.R.24) del CNR, C. so M. D'Azeglio, 42-10125 Torino (Italy).
\end{abstract}

(Received July 3, 1979)

\begin{abstract}
A complete discussion with an extension to the high temperature range of the main results of a recent theory ${ }^{1}$ on the electrical conduction and current noise in discontinuous metal films is given. In this theory the main conduction mechanism is a direct-tunnelling process of the electrons between metal islands within the insulator substrate. The tunnelling electrons must overcome a potential barrier which is temperature dependent. The model assumes that the electrons trapped in surface states can tunnel in the metal states giving rise to a double charge layer at the interface. The current noise is generated by a modulation mechanism of the carrier direct tunnelling due to thermal fluctuation of the potential barrier height, generated by a corresponding fluctuation of the surface charge.

Experimental results for the conductivity and current noise behaviour on Au film deposited on sapphire tube are also given up to a temperature of about $950 \mathrm{~K}$, and interpreted on the basis of the above theory.
\end{abstract}

\section{INTRODUCTION}

The electrical properties of discontinuous metal films are very different from those of the metal bulk. In particular they show: a) an anomalously large electrical resistivity, b) a negative thermal coefficient of resistivity (TCR) and c) the presence of an electrical current noise.

The discontinuous structure of the films of the type represented in Figure 1 strongly depends on the film deposition procedure. It is possible to vary the island dimensions within wide limits by evaporating the metal on a cooled or heated substrate. When the films are annealed at a high temperature, coalescence phenomena take place and the conductivity properties become mainly related to little gaps between metal islands along preferred conduction channels. In this case percolation processes may play an important role.

In theories of electrical conduction on discontinuous metal films, a tunnelling mechanism between metal islands is generally assumed, particularly in the low temperature range. The difficulty with these theories is that discontinuous films generally show a large negative TCR, which cannot be explained by assuming a simple tunnelling process.

Neugebauer and $\mathrm{Webb}^{2}$ suggested a tunnelling activated process related to a charge transfer between initially neutral islands. The activation energy in this process arises from the electrostatic energy of the charged metal islands, and is inversely proportional to the island dimensions. The theory does not explain the large TCR observed, for instance, in 


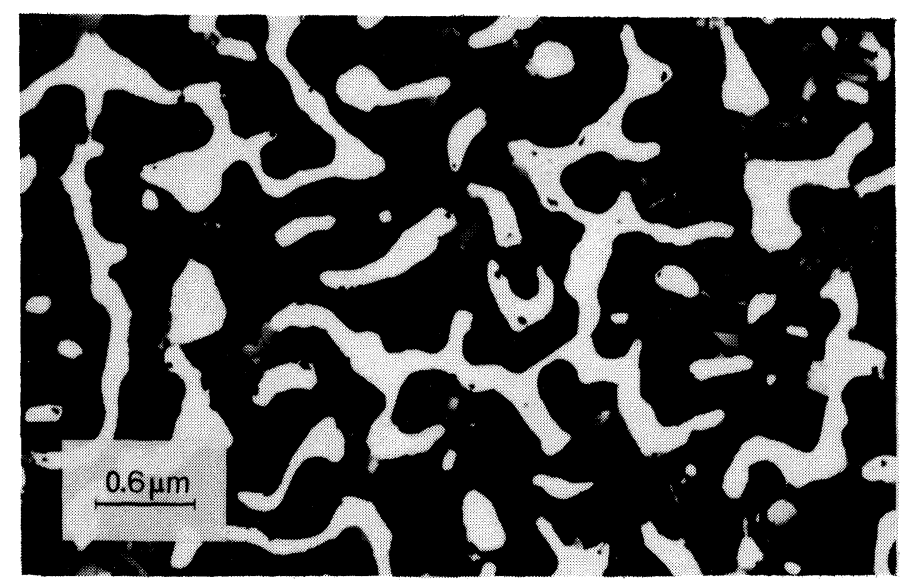

FIGURE 1 Electron micrograph of discontinuous Au film on sapphire substrate after annealing at $780 \mathrm{~K}$ in vacuum $\left(10^{-9}\right.$ mbar).

gold films having the large metal islands structure, obtained by annealing the film at about $780 \mathrm{~K}$.

A fundamental approach was given by $\mathrm{Hill}^{3}$ who assumed that the tunnelling path of the conduction electrons is through the insulating substrate. The conduction mechanism in Hill's approach was assumed to be enhanced by ion-traps, reducing both the barrier height and width, and giving rise to a thermally activated process, which explains the observed TCR of the film in a suitable range of temperature.

An improvement of this trap-assisted model is the multiple ion-trap hopping model proposed by Tick and Fehlner. ${ }^{4}$

Another possible mechanism is the thermionic emission which can contribute to the conduction process mainly in the highest temperature range.

Even if these theories can account for the electrical conduction in a rather restricted range of temperature, none of them provides the right order of magnitude for the current noise related to the spontaneous fluctuation of the film conductivity.

Williams and Burdett, ${ }^{5}$ were able to explain the $1 / \mathrm{f}$ behaviour of the electrical noise in discontinuous films, but their theory, as other theories based on the trap assisted tunnelling mechanism, cannot give the right order of magnitude of the current noise. ${ }^{6,7}$

In the present paper we discuss the main results of a recent theory which gives the right order of magnitude for the noise intensity, explains its power spectrum and also accounts for the conductivity vs. temperature behaviour in a temperature range much larger than in the previous theories $(25 \mathrm{~K}$ to $750 \mathrm{~K})$.

New results related to the conductivity and current noise behaviour up to $950 \mathrm{~K}$ on $\mathrm{Au}$ films deposited on sapphire tube substrate are given and discussed on the basis of the above theory. Possible improvements of the theory, as well as its application to other areas of interest (as, for instance, to the case of thick films, which show electrical characteristics very similar to those of discontinuous films ${ }^{6,7,8}$ ) are also discussed in the following sections.

\section{THEORETICAL MODEL}

The main concepts on which the model described in a previous paper ${ }^{1}$ is based, are:

a) The strong current noise observed in discontinuous films is actually a modulation noise of the type observed in semiconductors, where the stochastic change of the recombination centres, changing the recombination probability of electrons and holes, modulates the free carrier density and thus the conductivity of the semiconductor itself. In discontinuous films the mechanism of conductivity modulation is completely different and it is related to the fluctuation of the potential barriers existing in the gaps between metal islands.

b) The potential barrier height in the gaps depends on the position of the Germi level (F. 1) with respect to the conduction band of the insulator. Because electron tunnelling takes place near the surface, it is expected that surface states play an important role in determining the position of the F. 1. Statistical thermodynamics predicts a change in the position of the F.1. with temperature related to the 


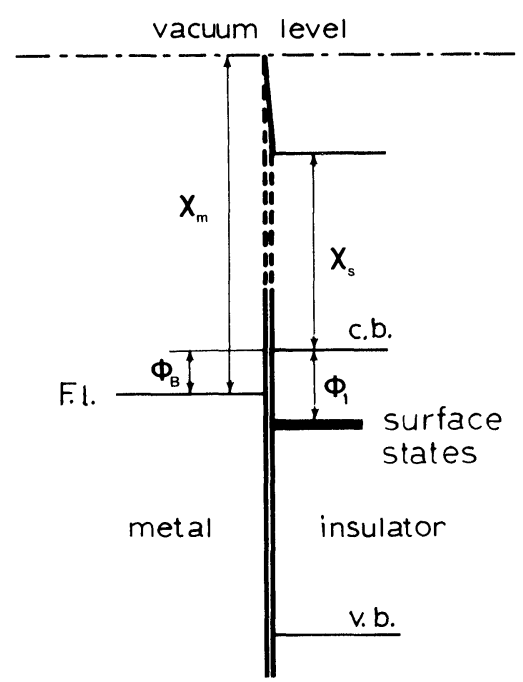

FIGURE 2 Energy diagram for the metal-island to substrate contact. $\chi_{m}$ and $\chi_{s}$ are the electron work functions for the metal and the insulator respectively, $\phi_{1}$ is the energy of the surface state level with respect to the conduction band of the insulator, and $\phi_{B}$ the potential barrier energy.

tunnelling of electrons from surface states into the metal islands. This could be the main mechanism responsible for the negative TCR of discontinuous metal films as quantitative calculations show. ${ }^{6,7,8}$ The potential barrier height fluctuations in this model arise from the statistical fluctuation of the number of ionized surface states. A sketch of the model is represented in Figure 2.

The temperature dependent barrier height $\phi_{B}(T)$ is self-consistently calculated by applying FermiDirac statistics to the electron system comprising the Fermi sea in the metal and the electrons trapped in surface states within the insulator. represented by a single highly degenerate level $\phi_{1}$ below the conduction band of the insulator itself.

The position of the level $\phi_{1}$ with respect to the Fermi level in the metal is determined by the surface charge $\gamma(T)$ created by the surface states ionization, with transfer of electrons to the metal by a tunneling process.

Detailed calculations are given by Celasco et al. ${ }^{1}$ The barrier $\phi_{B}(T)$ is given by the implicit equation given below:

$$
\begin{aligned}
& \phi_{B}(T)=\chi_{m}-\chi_{s}+\frac{e^{2} d}{12 \epsilon_{0} \epsilon_{r}^{*}} {\left[2 d\left(\frac{m^{*} k T}{2 \pi \hbar^{2}}\right)^{3 / 2} e^{-\phi_{B}(T) / k T}\right.} \\
&\left.-\delta e^{-\left[\phi_{2}-\phi_{B}(T)\right] / k T}\right]
\end{aligned}
$$

where $e$ is the electron charge, $d$ the spacing between metal islands, $\epsilon_{0}$ the vacuum permittivity, $\varepsilon_{r}^{*}=1 / 3 \epsilon_{r}$ the effective dielectric constant of the substrate, $m^{*}$ the effective electron mass at the bottom of the conduction band of the insulator, $k$ the Boltzmann's constant, $\hbar$ the reduced Planck's constant, $\delta$ the density of surface states. The other quantities are defined in Figure 2.

\section{ELECTRICAL CONDUCTION}

The behaviour of $\phi_{B}(T)$ with temperature, in the case of gold films on sapphire, is shown in Figure 3.

The electrical conductivity at each temperature between two metal islands is thus calculated by assuming a direct tunnelling process through the barrier $\phi_{B}(T)$. Several assumptions should be made concerning the gap width, the island dimensions and the tunnelling active surface for each island pair in order to get the absolute value of the conductance for the whole film. However the temperature dependence of this quantity turns out to have a typical behaviour which fits the experimental data well over a wide temperature range. In Figure 3 this quantity is shown for gold films on sapphire and compared with the experimental data in a range of temperatures from $77 \mathrm{~K}$ up to $750 \mathrm{~K}$.

It is interesting to note that in such a wide range of temperatures the Arrhenius plot of the conductivity is not the straight line that would be expected for a thermally activated process.

The tunnelling conductance $G_{1}$ in the low temperature range assumes the expression: ${ }^{1}$

$G_{1}=l(2 m)^{1 / 2}(e / h)^{2} \phi_{B}^{1 / 2}(T) \exp \left\{-\left[4 \pi(2 m)^{1 / 2} / h\right] d \phi_{B}^{1 / 2}(T)\right\}$,

where $l$ is the length of the active gap between metal islands, $m$ the electronic mass and $h$ is Planck's constant.

As seen in Figure 3, at temperatures above $200 \mathrm{~K}$ the barrier height $\phi_{B}(T)$ drops down to $0.1 \mathrm{eV}$, a value which excludes use of the approximate expressions for the tunnelling current given by Simmons, ${ }^{9}$ which require that $\phi_{B}(T) \gg k T$.

In this case Eq. (2) must be replaced by a more general expression which has been obtained by Celasco et al. ${ }^{8}$ This expression gives the same results as Eq. (2) in the case of low temperatures but represents a better approximation in the high temperature range. The conductivity curve 1 shown in Figure 3 is based on this new expression.

In the same figure the thermionic conductance, 


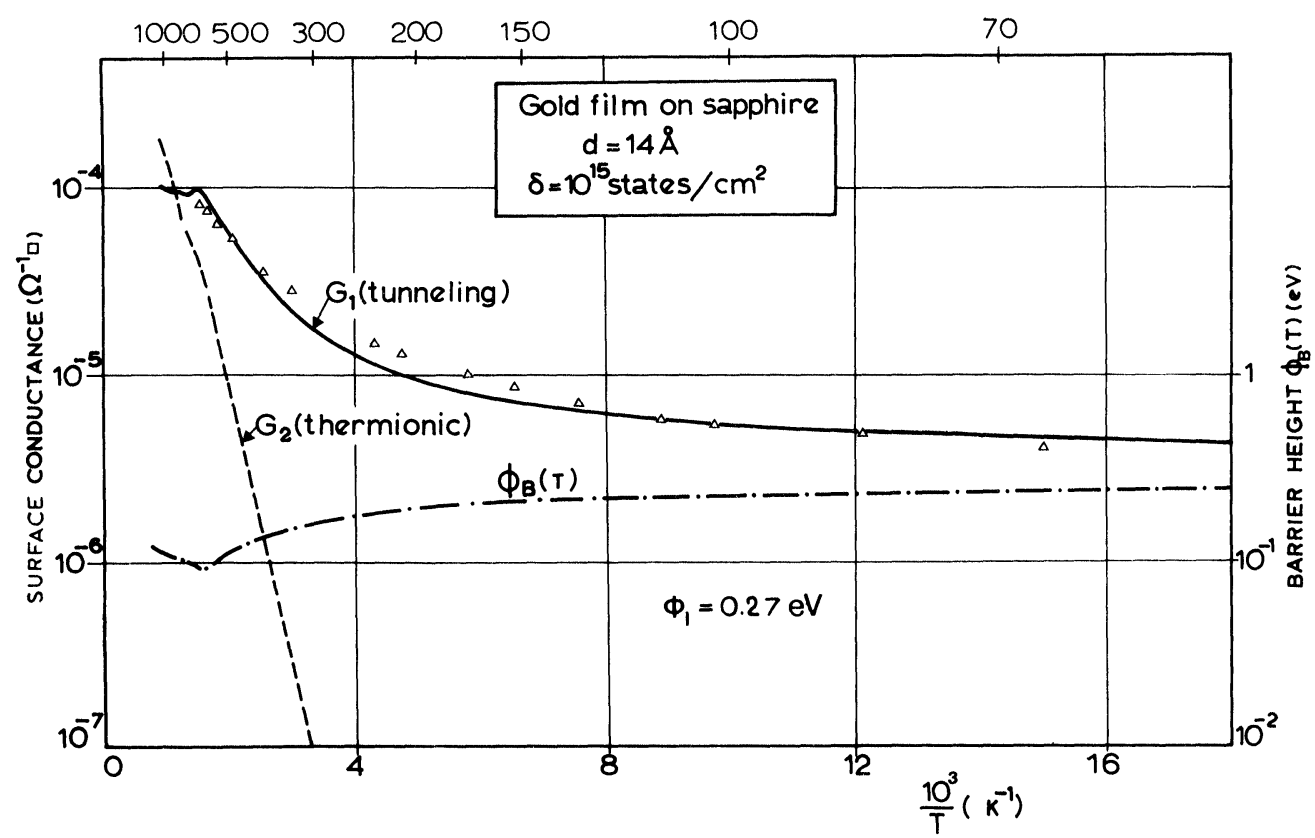

FIGURE 3 Logarithmic plot of the tunnelling and thermionic conductances, and of the barrier height $\phi_{B}(T)$ vs. $1 / T$ for an $A u$ film evaporated on sapphire substrate. The points are experimental, the theoretical curves are calculated as best fits of the experimental results by assuming $d=1.4 \times 10^{-9} \mathrm{~m}, \delta=10^{19} \mathrm{states} / \mathrm{m}^{2}, \phi_{1}=0.27 \mathrm{eV}$. and $l=3 \times 10^{-7} \mathrm{~m}\left[\right.$ After Celasco et $\left.\mathrm{al}^{8}\right]$.

curve 2, is given by the following expression:

$$
G_{2}=\operatorname{dl}\left(4 \pi m^{*} e k^{2} / h^{3}\right) T^{2} \exp \left[-\phi_{B}(T) / k T\right] .
$$

It is worth noting that with the choice of parameters reported in the caption of Figure 3, the thermionic component becomes comparable with the tunnelling component only at very high temperatures.

\section{CURRENT NOISE}

The origin of current noise is due to the fluctuation of the number $n(T)$ of ionized surface states around their average number $n_{0}(T)$.

In principle a normal fluctuation $\overline{\left(n-n_{0}\right)^{2}}=n_{0}$ could be assumed, but in order to take into account a feedback effect on the ionization which arises from the change of the surface charge density, the Nyquist theorem has been used by Celasco et $\mathrm{al}^{1}{ }^{1}$ to determine the barrier energy fluctuation in thermal equilibrium

The $1 / \mathrm{f}$ behaviour of the spectrum is directly obtained by taking into account the fact that an uniform distribution of the surface states gives a distribution of ionization times $T_{i}$ which, because of the exponential character of the tunnelling equation, ${ }^{1}$ is of the type

$$
P\left(\mathrm{~T}_{i}\right)=\frac{1}{\mathrm{~T}_{i}}
$$

Distributions of the type described by Eq. (4) are known to give $1 / \mathrm{f}$ spectra, and are used to explain $1 / \mathrm{f}$ noise in semiconductors. ${ }^{10}$

Both the intensity and the shape of the power spectrum of the current noise in discontinuous films obtained by this theory are consistent with experimental results.

In Figure 4 the experimental power spectra of the relative conductance noise $\left\langle\Delta G^{2}\right\rangle /\langle G\rangle^{2}$ for a gold film on sapphire substrate are reported for two different temperatures. The $1 / \mathrm{f}$ behaviour can be accounted for by a general expression worked out by Celasco et al: ${ }^{1}$

$$
\begin{aligned}
\psi_{F}(\omega) & =\frac{k T e^{2} \phi_{B}^{-1}(T)}{48 \pi \epsilon_{0} \epsilon_{r}^{*} l}\left[\frac{4 \pi(2 m)^{1 / 2} d}{h}-\phi_{B}^{-1 / 2}(T)\right]^{2}\left[\ln \frac{\mathrm{T}_{M}^{\prime}}{\mathrm{T}_{m}^{\prime}}\right] \\
& \times \frac{1}{\omega}\left[\tan ^{-1}\left(\omega \mathrm{T}_{M}^{\prime}\right)-\tan ^{-1}\left(\omega \mathrm{T}_{m}^{\prime}\right)\right] \frac{D^{2} L}{L^{\prime 3}}
\end{aligned}
$$




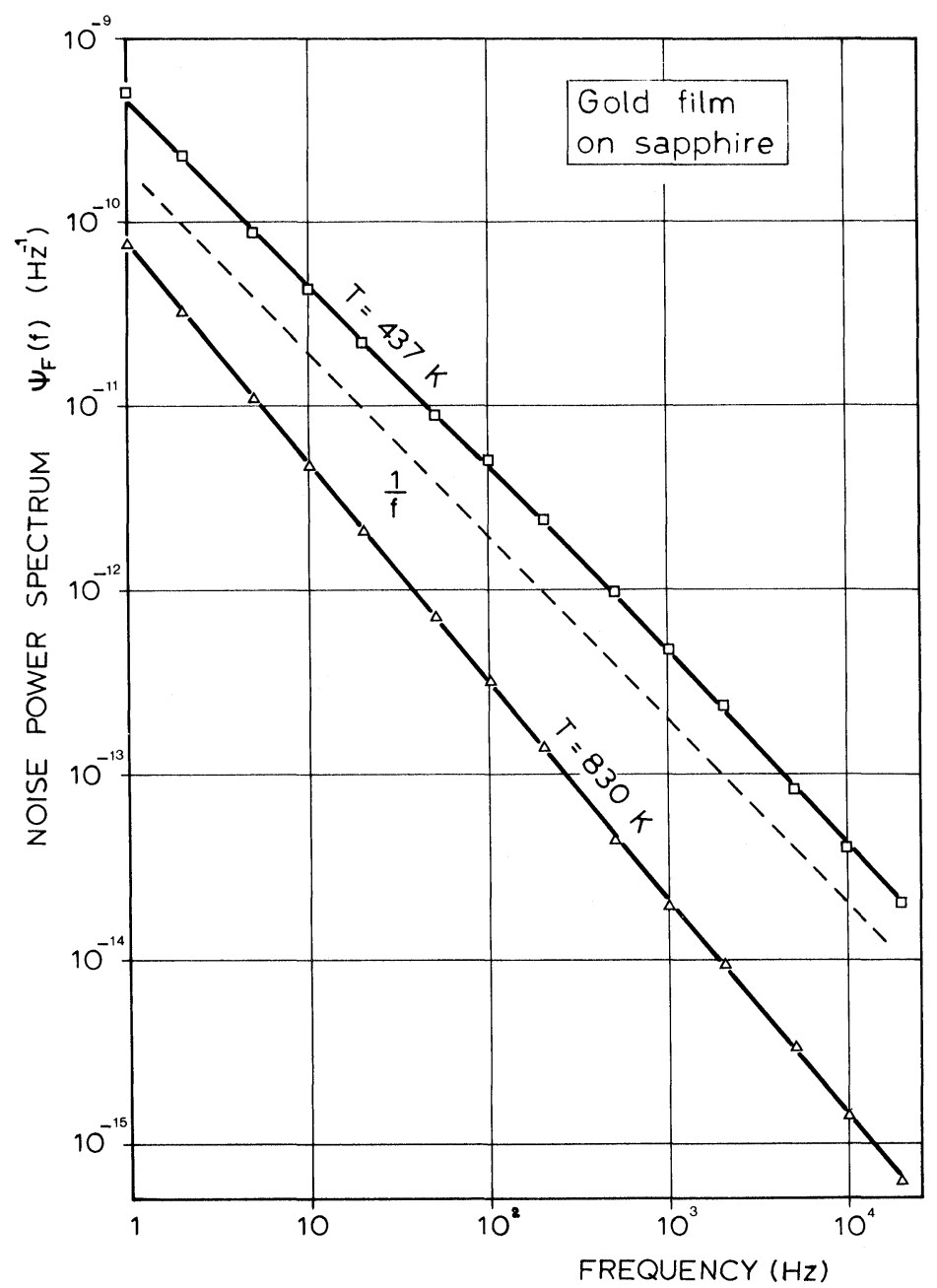

FIGURE 4 Experimental power spectra, at two different temperatures, of the relative conductance noise $\psi_{F}(f)$ in $\mathrm{Au}$ film evaporated on sapphire substrate in a vacuum of $10^{-9} \mathrm{mbar}$.

where $D$ is the average linear dimension of the metal island, $L$ and $L^{\prime}$ are respectively the width and the length of the film, $\mathbf{T}_{m}^{\prime}$ and $\mathbf{T}_{M}^{\prime}$ are constants related respectively to the shortest and the longest ionization times $T_{i}$ through the relation:

$$
\mathbf{T}_{i}^{\prime}=\frac{1}{1+\theta(T)} \cdot \mathbf{T}_{i}
$$

The quantity $\theta(T)$ represents the ratio between the ionization time $\mathrm{T}_{i}$ and the occupation time $\sigma_{i}$ and is a function of the temperature only.

The relative conductance noise $\left\langle\Delta G^{2}\right\rangle /\langle G\rangle^{2}$ is obtained by integrating Eq. (5) with respect to $\omega$ from $-\infty$ to $+\infty$.
After few minor approximations, the final expression is obtained:

$\frac{\left\langle\Delta G^{2}\right\rangle}{\langle G\rangle^{2}}=\frac{k T e^{2}}{12 \epsilon_{0} \epsilon_{r}^{*}} \frac{1}{l\langle G\rangle^{2}}\left(\frac{d G}{d \phi_{B}}\right)^{2} \frac{D^{2} L}{L^{\prime 3}}$.

The value of the derivative $d G / d \phi_{B}$ appearing in Eq. (7) has been calculated by Celasco et $\mathrm{al}^{1}$ using an approximation valid in the low temperature range:

$$
\frac{d G}{d \phi_{B}}=\frac{\phi_{B}^{-1 / 2}(T)}{2}\left[\frac{4 \pi(2 m)^{1 / 2}}{h} d-\phi_{B}^{-1 / 2}(T)\right]\langle G\rangle
$$

The theoretical results are in fair agreement with the experimental ones as reported by Celasco et al. ${ }^{1}$ 


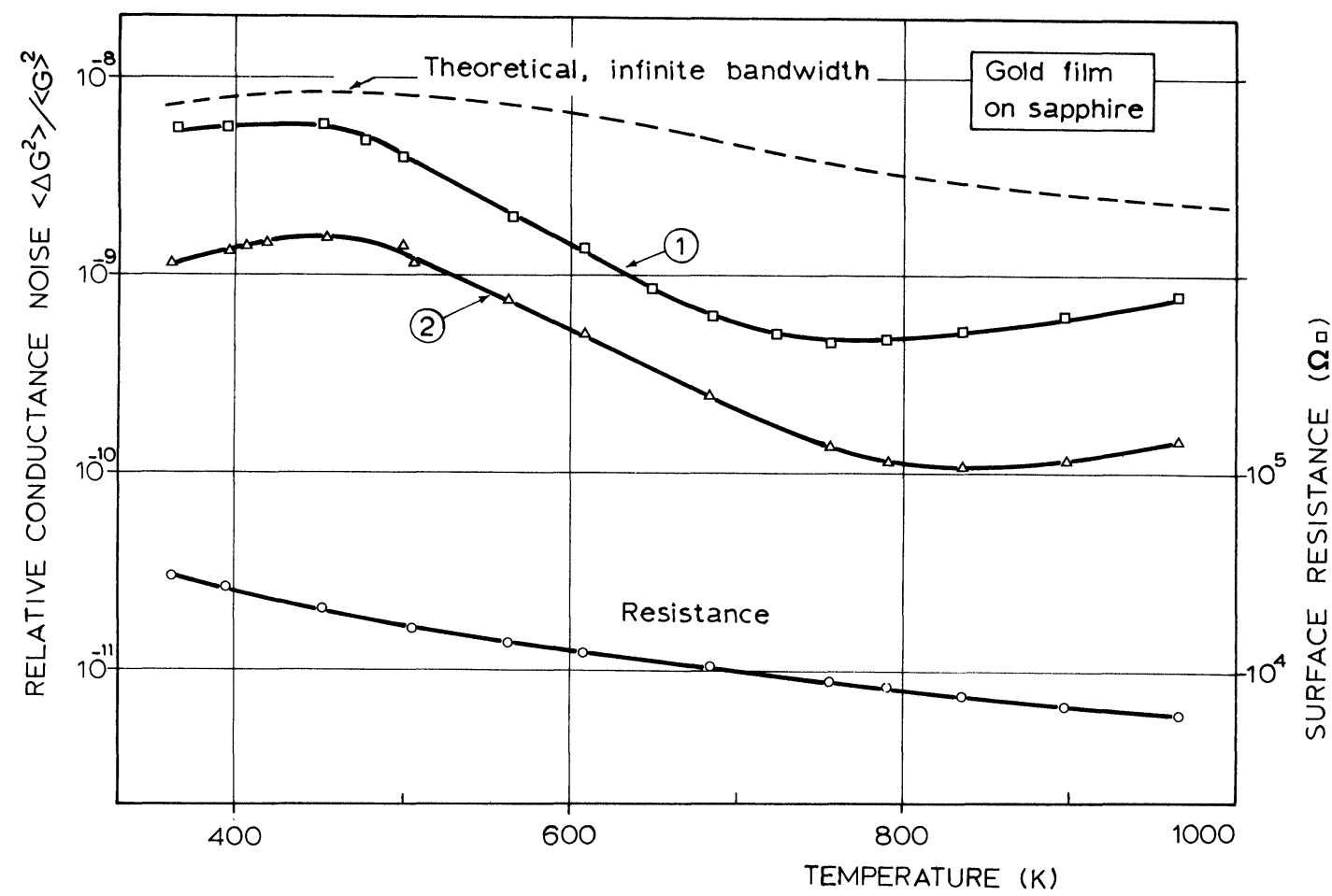

FIGURE 5 Relative conductance noise $\left\langle\Delta G^{2}\right\rangle /\langle G\rangle^{2}$ and resistance vs. temperature for an Au film evaporated at $620 \mathrm{~K}$ in a vacuum of $10^{-6} \mathrm{mbar}$. Film dimensions are $1.0 \mathrm{~mm}^{2} \times 1.0 \mathrm{~mm}^{2}$. The points are experimental. The broken line is computed from Eq. (14) of the paper by Celasco et al ${ }^{11}$ with $D=6 \times 10^{-7} \mathrm{~m}, l=2 \times 10^{-7} \mathrm{~m}, \phi_{B}(T)$ has been obtained from Eq. (1) with $\delta=10^{19}$ states $/ \mathrm{m}^{2}, \phi_{1}=0.25 \mathrm{eV}$ and $d=1.3 \times 10^{-9} \mathrm{~m}$. The integral noise has been detected in two different bands: (1) $1 \mathrm{~Hz}$ to $100 \mathrm{kHz}$, (2) $1 \mathrm{kHz}$ to $100 \mathrm{kHz}$.

An extension of the validity of Eqs. (7) and (8) to the high temperature range has been determined. ${ }^{11}$

A comparison between the experimental and the theoretical results is made in Figure 5.

As seen from this figure, the theory describes the main features of the noise intensity and gives the right order of magnitude for the noise for the values of the parameters involved. The agreement between experimental and theoretical results is however not completely satisfactory from the quantitative point of view.

The theoretical model should be considered to be an over simplification of reality. In particular the very complicated film structure has been replaced in the calculations by an array of islands having a size which is the average of the real one, separated by equal gaps, whose width and effective tunnelling cross-section were taken as fitting parameters. From the experimental point of view, current noise measurements at high temperatures are very difficult. In particular, in consideration of the results reported in Figure 5 for gold, it is worth noting that the increase of the power spectrum of the relative conductance fluctuation may be related to slow irreversible structural changes and to the evaporation of the film. Further experiments are needed to clarify this point.

\section{CONCLUSIONS}

In this paper the main lines of a new theory on the electrical conduction in discontinuous metal films have been sketched and compared with typical experimental results. The theory explains the temperature behaviour of the conductivity in an extended temperature range without assuming a direct thermally activated process for the free carrier transport between metal islands. In the last case (trap-assisted tunnelling, thermally activated tunnelling etc.) one would expect that the Arrhenius plot of the conductivity vs. temperature becomes a straight line, contrary to the experimental results. Furthermore the theory gives the right order of 
magnitude of the current noise, while preceding theories yield a value many orders of magnitude below the experimental value. Many improvements of the theory are possible.

In particular the theory should introduce suitable distribution functions for the width and the effective tunnelling cross-section of the metal islands, and should treat the conductivity as a percolation process (see Forlani and Prudenziati ${ }^{12}$ for thick film resistors).

Application of the above theory to thick film resistors, whose structure is similar to that of the discontinuous films being constituted of metallic particles separated by small gaps filled by an insulating oxide, seems in principle possible.

Chen ${ }^{13}$ has recently proposed a mechanism very similar to that developed in the theory discussed above explain current noise in these films. An accurate study of the noise intensity and power spectrum vs. temperature would be needed to have a better understanding of the real mechanism of electrical conduction taking place in thick films.

\section{REFERENCES}

1. M. Celasco, A. Masoero, P. Mazzetti and A. Stepanescu, Electrical conduction and current noise mechanism in discontinuous metal films. I. Theoretical, Phys. Rev. B. 17 (1978) 2553-63.
2. C. A. Neugebauer and M. B. Webb, Electrical conduction mechanism in ultrathin evaporated metal films, J. Appl. Phys. 33 (1962) 74-82

3. R. Hill, Electrical conduction in ultrathin metal films. I. Theoretical, Proc. Roy. Soc. A. 309 (1969) 377-95.

4. P. A. Tick and R. P. Fehlner, Electrical behaviour of composite discontinuous films, J. Appl. Phys. 43 (1972) 362-68.

5. J. L. Williams and R. K. Burdett, Current noise in thin gold films, J. Phys. C, 2 (1969) 298-306.

6. M. Celasco, A. Masoero, P. Mazzetti and A. Stepanescu, Electrical conduction and current noise mechanism in discontinuous metal films. II. Experimental Phys. Rev. B 17 (1978) 2564-74.

7. M. Celasco, A. Masoero and A. Stepanescu, Electrical conduction and current noise in discontinuous platinum films, Phys. Rev. B. 17 (1978) 3040-46.

8. M. Celasco, A. Masoero and A. Stepanescu, High temperature conductance of discontinuous metal films, Proc. 4th Int. Thin Films Congr. Loughborough (1978), Thin Solid Films. 57-1, (1979) 147-52.

9. J. C. Simmons, Generalized thermal J-V characteristic for the electric tunnel effect, J Appl. Phys. 35 (1964) 2655-58.

10. A. Van Der Ziel, Fluctuation Phenomena in SemiConductors, (Butterworths, London 1959).

11. M.. Celasco, A. Masoero and A. Stepanescu, The high temperature behavior of electrical noise in discontinuous metal films, Thin Solid Films 66 (1980) $111-10$

12. F. Forlani and M. Prudenziati, Electrical conduction by percolation in thick film resistors, Electrocomp. Sci. Technol., 3 (1976) 77-83.

13. T.M. Chen, Current noise in thick film resistors, in: D. Wolf (ed.), Noise in Physical Systems, Vol. 2. Springer-Verlag, Berlin, Heidelberg, New York (1978) 134-39. 

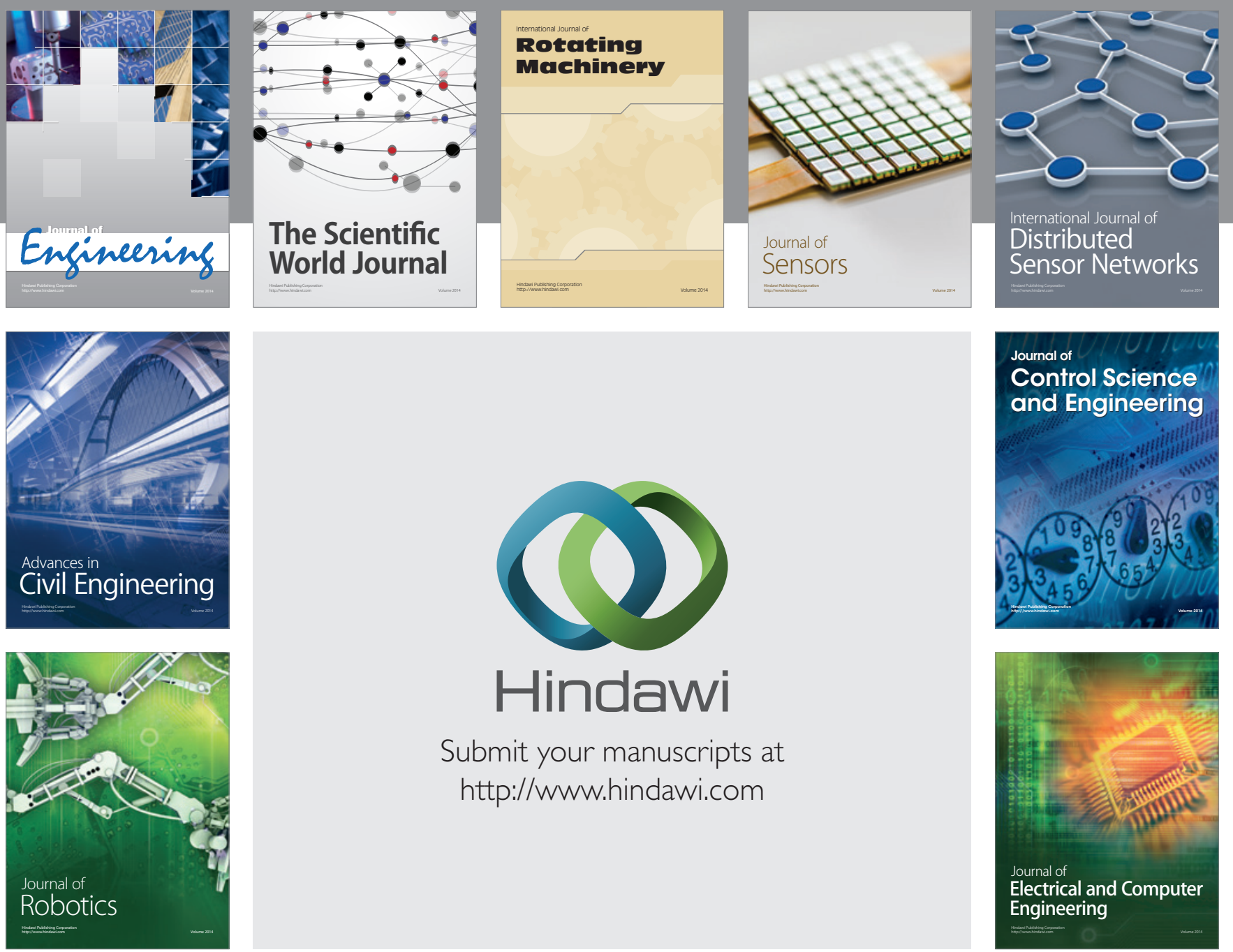

Submit your manuscripts at

http://www.hindawi.com
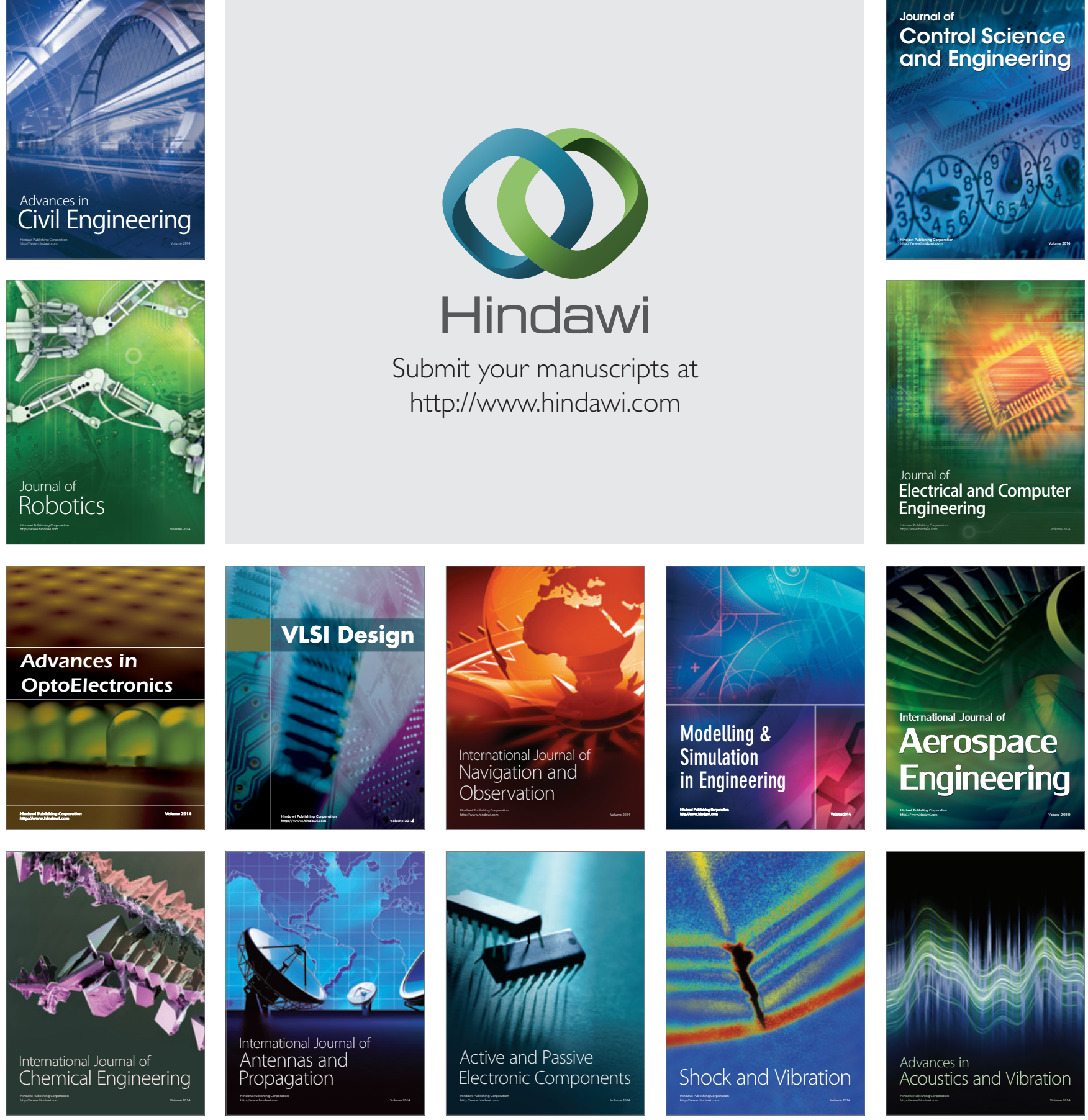